\title{
Estimation of Soil Macro Arthropods in Cotton Intercropped with Groundnut under Organic, Integrated and Conventional Farming Systems
}

\author{
Salavuddin Mohammad*, Bontha Rajasekar and R.K. Patil \\ Department of Agricultural Entomology, University of Agricultural Sciences, \\ Dharwad - 580 005, Karnataka, India \\ *Corresponding author
}

\begin{tabular}{|c|c|}
\hline & A B S T R A C T \\
\hline Keywords & $\begin{array}{l}\text { A field investigation was undertaken during Kharif- } 2013 \text { to estimate the } \\
\text { population of soil macro arthropods in cotton intercropped with groundnut under }\end{array}$ \\
\hline $\begin{array}{l}\text { Soil macro } \\
\text { arthropods, } \\
\text { Conventional } \\
\text { and pitfall traps. }\end{array}$ & $\begin{array}{l}\text { organic, integrated and conventional farming systems. Soil macro arthropod } \\
\text { consists of ants, scarabid beetles, centipedes, millipedes and others. Among the } \\
\text { different farming systems, organic farming recorded more population of total soil } \\
\text { macro arthropods ( } 147.64 / 15 \text { pitfall traps) followed by integrated farming system }\end{array}$ \\
\hline Article Info & (119.71/15 pitfall traps) and conventional farming system (91.90/15 pitfall traps). \\
\hline $\begin{array}{l}\text { Accepted: } \\
29 \text { April } 2017 \\
\text { Available Online: } \\
10 \text { May } 2017\end{array}$ & $\begin{array}{l}\text { Irrespective of farming systems the population of soil macro arthropods gradually } \\
\text { increased from first fortnight of June }(88.22 / 15 \text { pitfall traps) and reached to peak } \\
\text { population during second fortnight of August (190.56/15pitfall traps) and } \\
\text { gradually decreased to least population during second fortnight of December } \\
\text { (52.11/15pitfall traps). }\end{array}$ \\
\hline
\end{tabular}

\section{Introduction}

Soil is the most valuable resource, since this is essential to the entire plant life on which animal and human life depends. Soil contains wide assortment of organic, inorganic substances, water and gases thus provide a unique medium for growth and development of micro-organisms and soil invertebrates. In general, soil invertebrates are classified according to their size into three classes viz., micro fauna, meso fauna and macro fauna. Soil ecosystem services are reliant upon soil fauna which provide benefit to human population. They support most production systems through soil formation, nutrient cycling and primary production and also participate in provision of regulation services like climate regulation and detoxification. Without soil organisms, the soil would be a sterile medium that could not sustain crop production. Soil biota provides essential benefits for the functioning of agro ecosystems which are important for the long term sustainability of agriculture. They support essential soil processes and play a key role in maintaining the soil quality that is necessary for crop productivity. Soil organisms help to create and maintain beneficial soil structure and decomposing crop residues so that valuable nutrients are released for plant growth and contribute to soil carbon storage by mixing organic materials with mineral soil. Long-term and 
large number of soil inorganic fertilizer applications can affect negatively on soil fertility, soil biodiversity and crop products quality (Gruzdeva et al., 2007). By, considering the increasing importance of organic agriculture, soil organic manure applications are desirable and recommended. The effect deriving from organic manure applications includes numerous benefits resulting in an improvement of physical, biological and chemical soil properties, i.e. porosity, aggregates stability, water exchange and fertility (Tester, 1990). Heavy agricultural reliance on synthetic-chemical fertilizers and pesticides is having deleterious impacts on soil arthropods and the environment. With this background studies on soil arthropods in different farming systems were taken up.

\section{Materials and Methods}

The experiment was conducted during kharif 2013 at UAS Dharwad campus. The experiment was conducted with pitfall traps to know population of soil macro fauna which are moving above the ground. Pitfall traps are helpful in collecting the surface moving arthropods these are helpful to know the count of surface moving soil macro arthropods. Fifteen pitfall traps were placed in each field of agriculture, horticulture and forest ecosystems for collection of soil macro fauna.. Fifteen pitfall traps were placed in each field of cotton intercropped with groundnut under organic, integrated and conventional farming systems for collection of soil macro fauna. The trap consists of plastic cups (5 $\mathrm{cm}$ in dia $7 \mathrm{~cm}$ in height). The traps were buried in the ground with their rim leveled with the soil surface to facilitate wandering fauna to fall inside. Each cup was filled with $50 \mathrm{ml}$ of $75 \%$ ethyl alcohol as killing agent with bit of glycerol to prevent the evaporation of alcohol. Observations were taken with fifteen days interval starting from June till the harvest of the crops. Population of each group was recorded. Further these were separated as different taxonomic groups and preserved for further needful. Data were subjected to statistical analysis by two ways ANOVA.

\section{Results and Discussion}

Organic farming system was recorded significantly higher population of 10.83 centipedes /15 pitfall traps, 11.29 spiders /15 pitfall traps, 13.86 others (cockroach, grasshoppers, crickets)/15 pitfall traps and 147.64 total macro arthropods $/ 15$ pitfall traps. This system was followed by integrated farming system in which population of 8.98 centipedes /15 pitfall traps, 8.90 spiders $/ 15$ pitfall trap), 5.00 others (cock roach, grass hoppers, crickets /15 pitfall traps and 119.71 total macro arthropods $/ 15$ pitfall traps) and least population of 7.26 centipedes /15 pitfall traps, 6.67 spiders $/ 15$ pitfall traps and 91.90 total macro arthropods/15 pitfall traps were recorded in conventional farming system (Table 1). Centipedes population was gradually increased from first fortnight of June (5.00/15 pitfall traps) and attained maximum during first fortnight of August (16.88/15 pitfall traps) then gradually decreased and attained minimum population during second fortnight of December (2.44/15 pitfall traps). Interaction effect was found significant. Significantly highest centipede population was recorded in organic farming system during first fortnight of August (19.67/15 pitfall traps). Least population was recorded in conventional farming system during second fortnight of December (1.67/15 pitfall traps) (Table 1). Spiders population gradually increased from first fortnight of June (6.88/15 pitfall traps) and attained maximum population during second fortnight of August (15.00/15 pitfall traps) then gradually decreased and attained minimum population during second fortnight of December (3.22/15 pitfall traps) (Table 1). 
Interaction effect was found significant. Significantly highest spiders population was found in organic farming system with second fortnight of August (18.00/15 pitfall traps) and significantly least population was in conventional farming system during second fortnight of December (2.00/15 pitfall traps) (Table 1). Population of other arthropods gradually increased from first fortnight of June (10.22/15 pitfall traps) and attained maximum population during second fortnight of July (17.55/15 pitfall traps) then gradually decreased and attained minimum population during second fortnight of December (5.88/15 pitfall traps). Interaction effect between different farming systems and dates of collection of arthropods did not differ significantly (Table 1). Population of total micro arthropods gradually increased from first fortnight of June (88.22/15 pitfall traps) and attained maximum during second fortnight of August (190.56/15 pitfall traps) then gradually decreased and attained minimum during second fortnight of December (52.11/15 pitfall traps) (Table 1). The interaction effect was also found significant. Significantly highest total macro arthropods was found in organic farming system during second fortnight of August (230.33/15 pitfall traps) and least was in conventional farming system during second fortnight of December (30.33/15 pitfall traps) (Table 1).

Irrespective of crops total soil macro fauna recorded was more in organic farming system followed by integrated and conventional farming systems. Total soil macro fauna comprised of ants, carabid beetles, spiders, centipedes, scarabids, millipedes. Most of the soil macro fauna are predatory in habit so, these predators are density dependent when the meso arthropods population is more automatically that will help to increase the soil macro arthropods. It may be also due to abundance of organic matter, microbial biomass, optimum moisture, soil temperature and food availability. Present findings are in corroborating with Shilpa et al. (2013), they have also recorded higher macro fauna in $20 \mathrm{t}$ of FYM per hectare treated plots along with native soil fauna. This may be due to abundant ant nests associated with ample food sources (organic matter, microbial biomass and prey etc), optimal soil moisture and temperature regimes in the soil and it may also be due to feeding habit, host preference and favorably altered microclimate during crop growth stages.

Present findings corroborate with the observations of Minarro et al., (2009) they also reported that ground beetles (Carabids), rove beetles (Staphylinidae), ants (Formicidae) and spiders (Araneae) sampled monthly with pitfall traps in the apple orchard a total of 4978 individuals collected. Carabids (56.8\% of the total catches) were the most abundant taxonomic group, followed by spiders $(20.7 \%)$, ants $(14.8 \%)$ and rove beetles $(7.7 \%)$. Present findings are also in line with Braman and Pendley (1993), Naureen et al., (2010) they were also concluded that the deterioration of macroinvertebrates population was less evident in organically managed fields compared to high input farming practices. The peak population occurred when there was sufficient rainfall, sunshine hours, food availability with optimum soil moisture and preferable optimum soil temperature during cropping period and also it may be due to the availability of suitable food (prey, vegetation etc).

Present findings are in close agreement with Abilasha et al., (2013b) who reported that fluctuation and gradual increase in the abundance of the soil macro-fauna from BAT (Before application of treatments) was noticed in baby corn ecosystem. Highest population was noticed during 75 DAG. 
Table.1 Population of soil macro arthropods in cotton Intercropped with groundnut under organic, integrated and conventional farming system during 2013 (Per 15 Pitfall traps)

\begin{tabular}{|c|c|c|c|c|c|c|c|c|c|c|c|c|c|c|c|c|}
\hline \multirow{2}{*}{$\begin{array}{c}\begin{array}{c}\text { Date of } \\
\text { collection(DC) }\end{array} \\
\text { Farming } \\
\text { systems (FS) }\end{array}$} & \multicolumn{4}{|c|}{ Centipedes (No./15 pitfall traps) } & \multicolumn{4}{|c|}{ Spiders (No./15 pitfall traps) } & \multicolumn{4}{|c|}{$\begin{array}{l}\text { Others (cockroach, crickets and } \\
\text { grasshoppers)(No./15 pitfall traps) }\end{array}$} & \multicolumn{4}{|c|}{$\begin{array}{c}\text { Total macro arthropods (No./15 pitfall } \\
\text { traps) }\end{array}$} \\
\hline & $\mathbf{O F}$ & IF & $\mathbf{C F}$ & Mean & OF & IF & $\mathbf{C F}$ & Mean & OF & IF & $\mathbf{C F}$ & Mean & OF & IF & $\mathbf{C F}$ & Mean \\
\hline June I fortnight & $\begin{array}{l}7.00 \\
(2.74)\end{array}$ & $\begin{array}{l}5.00 \\
(2.35)\end{array}$ & $\begin{array}{l}3.00 \\
(1.87)\end{array}$ & $5.00 \mathrm{k}$ & $\begin{array}{c}9.67 \\
(3.19)\end{array}$ & $\begin{array}{c}7.00 \\
(2.74)\end{array}$ & $\begin{array}{c}4.00 \\
(2.12)\end{array}$ & $6.88 \mathrm{~h}$ & $\begin{array}{l}13.67 \\
(3.76)\end{array}$ & $\begin{array}{l}10.00 \\
(3.24)\end{array}$ & $\begin{array}{c}7.00 \\
(2.74)\end{array}$ & $10.22 \mathrm{i}$ & $\begin{array}{l}117.67 \\
(10.87)\end{array}$ & $\begin{array}{l}86.00 \\
(9.30)\end{array}$ & $\begin{array}{l}61.00 \\
(7.84)\end{array}$ & $88.22 k$ \\
\hline June II fortnight & $\begin{array}{l}11.00 \\
(3.39)\end{array}$ & $\begin{array}{c}8.67 \\
(3.03)\end{array}$ & $\begin{array}{c}7.00 \\
(2.74)\end{array}$ & $8.88 \mathrm{~h}$ & $\begin{array}{l}10.67 \\
(3.34)\end{array}$ & $\begin{array}{l}7.67 \\
(2.86)\end{array}$ & $\begin{array}{c}5.67 \\
(2.48)\end{array}$ & $8.00 \mathrm{f}$ & $\begin{array}{l}17.67 \\
(4.26)\end{array}$ & $\begin{array}{l}14.67 \\
(3.89)\end{array}$ & $\begin{array}{l}10.67 \\
(3.34)\end{array}$ & $14.33 \mathrm{e}$ & $\begin{array}{l}130.67 \\
(11.45)\end{array}$ & $\begin{array}{l}105.00 \\
(10.27)\end{array}$ & $\begin{array}{l}84.00 \\
(9.19)\end{array}$ & $106.56 \mathrm{i}$ \\
\hline July I fortnight & $\begin{array}{l}12.67 \\
(3.63)\end{array}$ & $\begin{array}{l}12.00 \\
(3.54) \\
\end{array}$ & $\begin{array}{c}9.67 \\
(3.19) \\
\end{array}$ & $11.44 d$ & $\begin{array}{l}15.00 \\
(3.94)\end{array}$ & $\begin{array}{l}12.00 \\
(3.54) \\
\end{array}$ & $\begin{array}{l}10.00 \\
(3.24)\end{array}$ & $12.33 b$ & $\begin{array}{l}15.00 \\
(3.94) \\
\end{array}$ & $\begin{array}{l}14.00 \\
(3.81) \\
\end{array}$ & $\begin{array}{l}11.00 \\
(3.39) \\
\end{array}$ & $13.33 f$ & $\begin{array}{l}138.33 \\
(11.78) \\
\end{array}$ & $\begin{array}{l}120.67 \\
(11.01) \\
\end{array}$ & $\begin{array}{l}102.67 \\
(10.16)\end{array}$ & $120.56 f$ \\
\hline July II fortnight & $\begin{array}{l}12.00 \\
(3.54)\end{array}$ & $\begin{array}{l}10.67 \\
(3.34)\end{array}$ & $\begin{array}{c}9.67 \\
(3.19)\end{array}$ & $10.77 \mathrm{e}$ & $\begin{array}{l}14.00 \\
(3.81)\end{array}$ & $\begin{array}{l}12.67 \\
(3.63)\end{array}$ & $\begin{array}{l}10.00 \\
(3.24)\end{array}$ & $12.22 \mathrm{~b}$ & $\begin{array}{l}19.00 \\
(4.42)\end{array}$ & $\begin{array}{l}18.00 \\
(4.30)\end{array}$ & $\begin{array}{l}15.67 \\
(4.02)\end{array}$ & $17.55 a$ & $\begin{array}{l}152.00 \\
(12.35)\end{array}$ & $\begin{array}{l}133.00 \\
(11.55)\end{array}$ & $\begin{array}{l}113.67 \\
(10.69)\end{array}$ & $132.89 \mathrm{e}$ \\
\hline Aug I fortnight & $\begin{array}{l}19.67 \\
(4.49)\end{array}$ & $\begin{array}{l}17.00 \\
(4.18)\end{array}$ & $\begin{array}{l}14.00 \\
(3.81)\end{array}$ & $16.88 \mathrm{a}$ & $\begin{array}{l}15.67 \\
(4.02)\end{array}$ & $\begin{array}{l}12.00 \\
(3.54)\end{array}$ & $\begin{array}{c}9.00 \\
(3.08)\end{array}$ & $12.22 \mathrm{~b}$ & $\begin{array}{l}16.33 \\
(4.10)\end{array}$ & $\begin{array}{l}17.00 \\
(4.18)\end{array}$ & $\begin{array}{l}14.00 \\
(3.81)\end{array}$ & $15.77 \mathrm{c}$ & $\begin{array}{l}223.67 \\
(14.97)\end{array}$ & $\begin{array}{l}174.67 \\
(13.24)\end{array}$ & $\begin{array}{l}131.00 \\
(11.47)\end{array}$ & $176.44 c$ \\
\hline Aug II fortnight & $\begin{array}{l}17.67 \\
(4.26)\end{array}$ & $\begin{array}{l}15.67 \\
(4.02)\end{array}$ & $\begin{array}{l}13.00 \\
(3.67)\end{array}$ & $15.44 b$ & $\begin{array}{l}18.00 \\
(4.30)\end{array}$ & $\begin{array}{l}15.00 \\
(3.94)\end{array}$ & $\begin{array}{l}12.00 \\
(3.54)\end{array}$ & $15.00 \mathrm{a}$ & $\begin{array}{l}18.00 \\
(4.30)\end{array}$ & $\begin{array}{l}14.67 \\
(3.89)\end{array}$ & $\begin{array}{l}12.67 \\
(3.63)\end{array}$ & $15.11 d$ & $\begin{array}{l}230.33 \\
(15.19)\end{array}$ & $\begin{array}{l}191.67 \\
(13.86)\end{array}$ & $\begin{array}{l}149.67 \\
(12.25)\end{array}$ & $190.56 a$ \\
\hline Sep I fortnight & $\begin{array}{l}18.00 \\
(4.30)\end{array}$ & $\begin{array}{l}15.00 \\
(3.94)\end{array}$ & $\begin{array}{l}12.00 \\
(3.54)\end{array}$ & $15.00 \mathrm{c}$ & $\begin{array}{l}14.00 \\
(3.81)\end{array}$ & $\begin{array}{l}11.67 \\
(3.49)\end{array}$ & $\begin{array}{c}8.67 \\
(3.03)\end{array}$ & $11.44 \mathrm{c}$ & $\begin{array}{l}19.00 \\
(4.42)\end{array}$ & $\begin{array}{l}16.00 \\
(4.06)\end{array}$ & $\begin{array}{l}15.00 \\
(3.94)\end{array}$ & $16.66 \mathrm{~b}$ & $\begin{array}{l}211.33 \\
(14.55) \\
\end{array}$ & $\begin{array}{l}179.67 \\
(13.42) \\
\end{array}$ & $\begin{array}{l}145.00 \\
(12.06) \\
\end{array}$ & 178.67b \\
\hline Sep II fortnight & $\begin{array}{l}12.00 \\
(3.54)\end{array}$ & $\begin{array}{c}9.00 \\
(3.08)\end{array}$ & $\begin{array}{c}8.67 \\
(3.03) \\
\end{array}$ & $9.88 f$ & $\begin{array}{l}12.67 \\
(3.63)\end{array}$ & $\begin{array}{c}9.67 \\
(3.19)\end{array}$ & $\begin{array}{c}7.67 \\
(2.86) \\
\end{array}$ & $10.00 d$ & $\begin{array}{l}14.67 \\
(3.89) \\
\end{array}$ & $\begin{array}{l}12.00 \\
(3.54)\end{array}$ & $\begin{array}{l}10.67 \\
(3.34)\end{array}$ & $12.44 \mathrm{~g}$ & $\begin{array}{l}178.67 \\
(13.39) \\
\end{array}$ & $\begin{array}{r}149.33 \\
(12.24) \\
\end{array}$ & $\begin{array}{l}118.33 \\
(10.90) \\
\end{array}$ & 148.78d \\
\hline Oct I fortnight & $\begin{array}{l}11.00 \\
(3.39)\end{array}$ & $\begin{array}{l}10.00 \\
(3.24)\end{array}$ & $\begin{array}{c}7.00 \\
(2.74)\end{array}$ & $9.33 \mathrm{~g}$ & $\begin{array}{l}10.00 \\
(3.24)\end{array}$ & $\begin{array}{c}8.67 \\
(3.03)\end{array}$ & $\begin{array}{c}6.67 \\
(2.68)\end{array}$ & $8.44 \mathrm{e}$ & $\begin{array}{l}12.67 \\
(3.63)\end{array}$ & $\begin{array}{l}11.00 \\
(3.39)\end{array}$ & $\begin{array}{c}9.00 \\
(3.08)\end{array}$ & $10.88 \mathrm{~h}$ & $\begin{array}{l}157.33 \\
(12.56)\end{array}$ & $\begin{array}{l}100.33 \\
(10.04)\end{array}$ & $\begin{array}{l}98.00 \\
(9.92)\end{array}$ & $118.56 \mathrm{~g}$ \\
\hline Oct II fortnight & $\begin{array}{l}10.00 \\
(3.24)\end{array}$ & $\begin{array}{c}8.00 \\
(2.92)\end{array}$ & $\begin{array}{c}7.67 \\
(2.86)\end{array}$ & $8.55 \mathrm{i}$ & $\begin{array}{c}8.67 \\
(3.03)\end{array}$ & $\begin{array}{c}7.00 \\
(2.74)\end{array}$ & $\begin{array}{c}5.00 \\
(2.35)\end{array}$ & $6.88 \mathrm{~h}$ & $\begin{array}{l}10.00 \\
(3.24)\end{array}$ & $\begin{array}{c}8.00 \\
(2.92)\end{array}$ & $\begin{array}{c}7.00 \\
(2.74)\end{array}$ & $8.33 j$ & $\begin{array}{l}136.67 \\
(11.71)\end{array}$ & $\begin{array}{l}121.00 \\
(11.02)\end{array}$ & $\begin{array}{l}85.67 \\
(9.28)\end{array}$ & $114.44 h$ \\
\hline Nov I fortnight & $\begin{array}{c}7.67 \\
(2.86)\end{array}$ & $\begin{array}{c}6.00 \\
(2.55)\end{array}$ & $\begin{array}{c}4.67 \\
(2.27)\end{array}$ & $6.11 \mathrm{j}$ & $\begin{array}{c}7.00 \\
(2.74)\end{array}$ & $\begin{array}{c}5.67 \\
(2.48)\end{array}$ & $\begin{array}{c}3.67 \\
(2.04)\end{array}$ & $5.44 \mathrm{j}$ & $\begin{array}{c}9.67 \\
(3.19)\end{array}$ & $\begin{array}{c}8.00 \\
(2.92)\end{array}$ & $\begin{array}{c}6.67 \\
(2.68)\end{array}$ & $8.11 \mathrm{j}$ & $\begin{array}{l}119.67 \\
(10.96)\end{array}$ & $\begin{array}{l}100.67 \\
(10.06)\end{array}$ & $\begin{array}{l}67.33 \\
(8.24)\end{array}$ & $95.89 \mathrm{j}$ \\
\hline Nov II fortnight & $\begin{array}{c}4.67 \\
(2.27)\end{array}$ & $\begin{array}{c}3.00 \\
(1.87)\end{array}$ & $\begin{array}{c}1.67 \\
(1.47)\end{array}$ & $3.11 \mathrm{~m}$ & $\begin{array}{l}10.00 \\
(3.24)\end{array}$ & $\begin{array}{c}7.00 \\
(2.74)\end{array}$ & $\begin{array}{c}5.00 \\
(2.35)\end{array}$ & $7.33 \mathrm{~g}$ & $\begin{array}{l}10.00 \\
(3.24)\end{array}$ & $\begin{array}{c}7.00 \\
(2.74)\end{array}$ & $\begin{array}{c}4.67 \\
(2.27)\end{array}$ & $7.22 \mathrm{k}$ & $\begin{array}{l}107.67 \\
(10.40)\end{array}$ & $\begin{array}{l}87.00 \\
(9.35)\end{array}$ & $\begin{array}{l}55.67 \\
(7.49)\end{array}$ & 83.441 \\
\hline Dec I fortnight & $\begin{array}{c}4.67 \\
(2.27)\end{array}$ & $\begin{array}{c}3.67 \\
(2.04)\end{array}$ & $\begin{array}{c}2.00 \\
(1.58)\end{array}$ & 3.441 & $\begin{array}{c}8.00 \\
(2.92)\end{array}$ & $\begin{array}{c}5.67 \\
(2.48)\end{array}$ & $\begin{array}{c}4.00 \\
(2.12)\end{array}$ & $5.88 \mathrm{i}$ & $\begin{array}{c}8.67 \\
(3.03)\end{array}$ & $\begin{array}{c}5.67 \\
(2.48)\end{array}$ & $\begin{array}{c}3.67 \\
(2.04)\end{array}$ & 6.001 & $\begin{array}{l}90.67 \\
(9.55)\end{array}$ & $\begin{array}{c}73.33 \\
(8.59)\end{array}$ & $\begin{array}{l}44.33 \\
(6.70)\end{array}$ & $69.44 m$ \\
\hline Dec II fortnight & $\begin{array}{c}3.67 \\
(2.04)\end{array}$ & $\begin{array}{c}2.00 \\
(1.58)\end{array}$ & $\begin{array}{c}1.67 \\
(1.47)\end{array}$ & $2.44 n$ & $\begin{array}{c}4.67 \\
(2.27)\end{array}$ & $\begin{array}{c}3.00 \\
(1.87)\end{array}$ & $\begin{array}{c}2.00 \\
(1.58)\end{array}$ & $3.22 \mathrm{k}$ & $\begin{array}{c}9.67 \\
(3.19)\end{array}$ & $\begin{array}{c}5.00 \\
(2.35)\end{array}$ & $\begin{array}{c}3.00 \\
(1.87)\end{array}$ & 5.881 & $\begin{array}{l}72.33 \\
(8.53)\end{array}$ & $\begin{array}{l}53.67 \\
(7.36)\end{array}$ & $\begin{array}{l}30.33 \\
(5.55)\end{array}$ & $52.11 n$ \\
\hline Mean & $10.83 a$ & $8.98 b$ & $7.26 \mathrm{c}$ & 9.02 & $11.29 \mathrm{a}$ & $8.90 b$ & $6.67 \mathrm{c}$ & 8.95 & 13.86a & $11.50 \mathrm{~b}$ & $9.33 \mathrm{c}$ & 11.56 & 147.64 & 119.71 & 91.90 & 119.75 \\
\hline $\begin{array}{l}\text { Farming Systems } \\
\text { (FS) }\end{array}$ & \multicolumn{2}{|c|}{0.03} & \multicolumn{2}{|c|}{0.07} & \multicolumn{2}{|c|}{0.02} & \multicolumn{2}{|c|}{0.07} & \multicolumn{2}{|c|}{0.03} & \multicolumn{2}{|c|}{0.08} & \multicolumn{2}{|c|}{0.08} & \multicolumn{2}{|c|}{0.22} \\
\hline $\begin{array}{c}\text { Date of } \\
\text { collection (DC) }\end{array}$ & \multicolumn{2}{|c|}{0.05} & \multicolumn{2}{|c|}{0.15} & \multicolumn{2}{|c|}{0.05} & \multicolumn{2}{|c|}{0.14} & \multicolumn{2}{|c|}{0.06} & \multicolumn{2}{|c|}{0.17} & \multicolumn{2}{|c|}{0.17} & \multicolumn{2}{|c|}{0.48} \\
\hline FSXDC & \multicolumn{2}{|c|}{0.09} & \multicolumn{2}{|c|}{0.27} & \multicolumn{2}{|c|}{0.09} & \multicolumn{2}{|c|}{0.25} & \multicolumn{2}{|c|}{0.11} & \multicolumn{2}{|c|}{ NS } & \multicolumn{2}{|c|}{0.30} & \multicolumn{2}{|c|}{0.83} \\
\hline
\end{tabular}

OF - Organic farming $\quad$ IF - Integrated farming $\quad$ CF - Conventional farming $\quad$ Figures in the parenthesis are $\sqrt{\mathrm{x}}+05$ transformed values $\quad$ NS - Non-significant 
The present findings are in close agreement with the observations of Shilpa et al., (2013) reported treatments with application of FYM supported higher macro fauna and Umadevi et al., (2013) reported higher macro faunal activity was observed on 45 DAG (53.99/trap). Absence of chemicals, no or low levels of soil disturbance and eco friendly management techniques in organic fields which completely or partially absent in conventional managed fields, resulted in the buildup of beneficial arthropods (Mader et al., 2002). In conventional farming less population of micro arthropods was recorded. It may be due to intensive agriculture and excessive use of agrochemicals have resulted in an impoverished wild life especially reduced arthropod diversity and density in agriculture ecosystem. Because of high input fertilizers and pesticides a severe decline of biological diversity has been observed (Letourneau and Bothwell, 2008). The results also in line with the Patil et al., (2013), Ayuke et al., (2004), Santigo et al., (2009), Abbas and Parwez (2012) who have also reported that abundance of total micro arthropod population four times higher in organic farming system than compared to conventional farming system. Harsh climatic conditions could lead to gradual losses of micro arthropods.

\section{References}

Ayuke, F. O., Opando-Mbai, M. L., Rao, M. R. and Swift, M. J., 2004, An assessment of biomass transfer from green manure to soil macrofauna in agro-ecosystem soil macrofauna biomass. In: Batino, A. (Eds.), Managing nutrient cycles to sustain soil fertility in Sub-Saharan Africa, ASP, Nairobi, Kenya., 4: 65-76.

Abbas, M. J. and Parwez, H., 2012, Impact of edaphic factors on the diversity of Soil microarthropods in an agricultural ecosystem at Aligarh, Indian $J$. Fundamental and Appl. Life Sci., 2(1):185-191.

Abilasha, C. R., Kumar, N. G., Narasa Reddy, G., Shilpa V. Akkur and Mahabob Shah Sultani, 2013b, Changes in the soil meso-fauna in response to the application of various doses of farm yard manure in baby corn cropping system. $10^{\text {th }}$ National Symposium on Soil Biol. Ecol., p. 103.

Braman, S. K. and Pandley, A. F., 1993, Relative and seasonal abundance of beneficial arthropods in centipede grass as influenced by management practice. J. Econ. Ent., 86(2): 494-504.

Gruzdeva, L. I., Matveeva, E. M. and Kovalenko, T. E., 2007, Changes in soil nematode communities under the impact of fertilizers. Eurasian Soil Sci., 40: 681-693

Letourneau, D. K. and Bothwell, S. G., 2008, Comparision of organic and conventional farms, challenging ecologists to make biodiversity functional. Front. Ecol. Environ., 6: 430-438.

Mader, P., Fliessbach, A., Dubois, L., Gunst, F., and Niggilo, O., 2002, Soil fertility and biodiversity in organic farming. Sci., 296:1694-1697.

Minarro, M., Espadaler, X., Melero, V. X. and Suarez Alvarez, V., 2009, Organic versus conventional management in an apple orchard: effects of fertilization and tree-row management on grounddwelling predaceous arthropods, Agril. Forest Entomol., 11(2): 133-142.

Naureen, R., Rana, S. A., Khan, H. A. and Sohail, A., 2010, Assessment of possible threats to soil macroinvertebrates diversity in wheat fields from high input farming. Int. J. Agric. Bio., 12(6): 801-808.

Patil, R. K, Babalad, H. B. and Sanjaya 
Topagi., 2013, Soil arthropods population in different farming practices. $10^{\text {th }}$ National Symposium on soil Biol. Ecol., p. 65 .

Santiago, F., Peredo, P., Esperanza Parada, Z., Marcela Vega C., Claudia, P. and Barrera, S., 2009, Edaphic mesofauna community structure in organic and conventional management of cranberry (Vaccinium sp.) plantations: an agroecological approach. J. Soil. Sci. Pl. Nut., 9(3): 236-244.

Shilpa, V. Akkur., Kumar, N. G., Narasa Reddy, G. and Abilasha, C. R., 2013,
Abundance of soil macro-fauna in soybean ecosystem. $10^{\text {th }}$ National Symposium on Soil Biol. Ecol., pp. 112. Tester, C. F., 1990, Organic amendment effects on physical and chemical properties of a sandy soil. Soil Sci. Soc. Am. J., 65: 1284-1292.

Umadevi, Ngangom, Kumar, N. G., Basavaraj, P. K. and Narasa Reddy, G., 2013, Effect of higher dose of inorganic fertilizer on the soil macrofaunal population in maize ecosystem. $10^{\text {th }}$ National Symposium on Soil Biol. Ecol., p. 131.

\section{How to cite this article:}

Salavuddin Mohammad, Bontha Rajasekar and Patil, R.K. 2017. Estimation of Soil Macro Arthropods in Cotton Intercropped with Groundnut under Organic, Integrated and Conventional Farming Systems. Int.J.Curr.Microbiol.App.Sci. 6(6): 2424-2429. doi: https://doi.org/10.20546/ijcmas.2017.606.287 